\title{
Breast Imaging Devices for Nuclear Medicine*
}

\author{
Jennifer Prekeges, CNMT \\ Nuclear Medicine Technology, Bellevue College, Bellevue, Washington
}

Breast cancer has long been a significant cause of morbidity and mortality. Mammography is the first-line imaging examination used to detect breast cancer; it has high sensitivity but only moderate specificity. The currently used secondary imaging modalities, sonography and MRI, cannot weed out all the falsepositive lesions that mammography identifies as potentially malignant. Further, many patients do not image well on mammography, so there is a significant need for alternative imaging methods. Recently, technologies using small-field, pixelated detectors optimized for breast imaging have become available for both single-photon-emitting and positron-emitting radiopharmaceuticals. This article addresses the construction and functionality of several detector types and their application to imaging of the breast. Technical aspects of nuclear breast imaging will be discussed briefly. The article concludes with an assessment of the position of nuclear medicine imaging of the breast within the overall diagnostic scheme for breast cancer detection.

Key Words: breast imaging; breast cancer; pixelated imaging devices; semiconductor radiation detector

J Nucl Med Technol 2012; 40:71-78

DOI: $10.2967 /$ jnmt.111.097410

\section{B} reast cancer is a major cause of morbidity and mortality among women worldwide. Radiologic imaging is of primary importance in identifying and assessing breast tumors. Screening mammography identifies suggestive areas, and focused mammographic images are used for better characterizing them. However, mammography misses breast cancers in patients with breast implants, dense breasts, and other situations. In addition, mammography has a high falsepositive rate, requiring further imaging before proceeding to biopsy (1).

Sonography and MRI are the secondary imaging modalities generally used to further characterize breast lesions, but neither does so with high accuracy. Although MRI can be highly sensitive (up to 99\%), its specificity is around $25 \%$ (2). Breast ultrasound has specificity of only $34 \%$ for

\footnotetext{
Received Aug. 24, 2011; revision accepted Oct. 17, 2011.

For correspondence or reprints contact: Jennifer Prekeges, Nuclear Medicine Technology, Bellevue College, 3000 Landerholm Circle SE, Bellevue, WA 980076484.

E-mail: jennifer.prekeges@bellevuecollege.edu

Published online May 4, 2012.

*NOTE: FOR CE CREDIT, YOU CAN ACCESS THIS ACTIVITY THROUGH THE SNM WEB SITE (http://www.snm.org/ce_online) THROUGH JUNE 2014. COPYRIGHT @ 2012 by the Society of Nuclear Medicine, Inc.
}

breast cancers (2). Both modalities lead to unnecessary biopsies, and both may miss lesions in dense breasts. In addition, as many as $15 \%$ of patients referred for breast MRI cannot tolerate or refuse to undergo the procedure (3).

Because of these limitations, a potential role for nuclear medicine in breast cancer diagnosis exists. But neither standard $\gamma$-cameras nor PET tomographs are well suited to breast imaging. This continuing education article addresses new imaging devices that have been developed to provide more detailed nuclear medicine images of breast tissue.

\section{NUCLEAR BREAST IMAGING WITH CONVENTIONAL DEVICES}

Researchers have known from early on that the myocardial imaging agent ${ }^{99 \mathrm{~m}} \mathrm{Tc}$-sestamibi is taken up in breast tumors $(4,5)$. Uptake of this radiopharmaceutical in malignant tissues depends on blood flow, metabolic activity, and membrane potential (6). Sestamibi imaging was noted to be particularly helpful in patients with dense breast tissue, breast implants, a palpable mass with negative mammography findings, or a history of prior breast surgery (1).

Positron-emitting radiopharmaceuticals have likewise demonstrated potential for identifying and characterizing breast cancers. ${ }^{18} \mathrm{~F}-\mathrm{FDG}$, a mainstay of current oncologic practice, shows uptake in many breast tumors, especially those that are more advanced (6). ${ }^{18} \mathrm{~F}$-fluoroestradiol is an estrogen analog that is used to indicate the estrogen status of a breast cancer. ${ }^{18} \mathrm{~F}$-fluoro-L-thymidine uptake reflects cell proliferation, a primary indicator of tumor aggressiveness, and may be an excellent marker of response to therapy (7).

We can see from the above discussion that nuclear medicine has much to contribute to the detection of breast cancer in routine nuclear medicine and in the PET arena. The problem lies in imaging the breast using conventional cameras. Both $\gamma$-cameras and PET tomographs have significant drawbacks when it comes to imaging of the breast.

Breast imaging with ${ }^{99 \mathrm{~m}} \mathrm{Tc}$-sestamibi and a $\gamma$-camera was originally accomplished with prone-dependent imaging (1). On an imaging table with cut-outs, the patient was instructed to lie prone such that one breast fit into the cutout. The $\gamma$-camera was positioned laterally, as close to the patient's body as possible. But this positioning was still several centimeters away from the breast tissue, leading to decreased spatial resolution and an inability to see small lesions. Supine positioning potentially decreases the distance between breast and camera but causes the breast tissue to spread out over the chest wall, making it difficult to 
distinguish uptake in the breast from uptake in other tissues, notably liver and myocardium. Therefore, it is impossible to get a view of the breast that is both close to the detector and isolated from other tissues.

It would be preferable for comparison purposes to put the breast tissue directly on the camera face, in order to generate images in a format similar to that used in mammography. But this is not possible using standard $\gamma$-cameras. A $\gamma$-camera has considerable dead space around the periphery, because of the need for photomultiplier tubes (PMTs) outside the field of view (FOV) to pull detected $\gamma$-rays to their correct position, as required by Anger positioning logic. At least several centimeters of breast tissue closest to the chest wall are not in the FOV when one is trying to obtain a mammography-equivalent view with a standard $\gamma$-camera.

Additionally, the spatial resolution of a nuclear breast imaging device is crucial. The size of the primary lesion correlates directly with the likelihood that the cancer has spread. Because screening mammograms routinely show lesions that are $5 \mathrm{~mm}$ or less, nuclear medicine imaging must be able to find lesions in this size range. With a standard $\gamma$-camera and prone-dependent positioning, sensitivity for lesions less than $1 \mathrm{~cm}$ was low (1). To be useful in the arena of breast cancer, an imaging device must be capable of imaging subcentimeter-sized lesions.

PET of the breast with a standard PET tomograph suffers from similar drawbacks. Patients are typically supine for a PET scan, again spreading the breast tissue above the chest wall and creating the potential for interference by other organs that take up the radiopharmaceutical. Coregistered PET and CT images could assist in determining whether a lesion is located in the breast or in another tissue, but the resultant images are not directly comparable to a mammographic image. Conventional PET tomographs have spatial resolution of about $5 \mathrm{~mm}$, which again is borderline for early detection of breast tumors.

\section{COMMON FEATURES OF BREAST IMAGING DEVICES}

Nuclear medicine breast imaging has become increasingly popular over the last 5-10 y, thanks mainly to the development of cameras optimized for this purpose. Table 1 provides specifications for several commercially available systems. (More elaborate systems for nuclear breast imaging have been developed (8) but will not be discussed in this article.) Rather than discussing features of individual systems, we will review the features of these systems that make them suitable for breast imaging. The discussion relates primarily to imaging devices used with single-photon-emitting radiopharmaceuticals, followed by descriptions of 2 PET systems.

\section{Detector Material}

First, most of the new breast-imaging devices use detector materials other than thallium-activated sodium iodide (NaI (Tl)). Two manufacturers have systems using thallium- activated cesium iodide ( $\mathrm{CsI}(\mathrm{Tl}))$ scintillation crystals. Cesium iodide has a higher effective atomic number and higher mass density than $\mathrm{NaI}(\mathrm{Tl})$, giving it improved efficiency for absorbing high-energy $\gamma$-rays (9). It also releases more scintillation photons per megaelectron-volt of $\gamma$-ray energy. But the scintillation light is of a higher wavelength than is optimal for current PMTs, necessitating the use of avalanche photodiodes as the photon transducers. The scintillation decay time of $\mathrm{CsI}(\mathrm{Tl})$ is much longer than that of $\mathrm{NaI}$ (Tl), although this is not usually a problem for single-photon imaging. Cesium iodide crystals can be grown with a column microstructure in which each column acts as an optically isolated scintillator (9), fitting well with the idea of a pixelated detector system.

Several manufacturers are using a semiconductor material, namely cadmium zinc telluride (CZT), in breast imaging devices. Semiconductor radiation detectors eliminate some of the inefficiencies of the scintillation detection process by directly counting electrons produced by $\gamma$-ray interactions. Therefore, CZT detectors count many more electrons per radiation interaction and consequently have significantly improved energy resolution, compared with scintillation detectors $(5 \%-10 \%$ vs. $10 \%-15 \%)$. (The importance of energy resolution in breast imaging is debatable (10)). In addition, a 1-mm-thick semiconductor detector has about the same sensitivity for $140-\mathrm{keV}$ photons as does a 10-mm-thick scintillation detector (11), a plus in the design of a small-field imaging device.

\section{Pixelated Architecture}

A second major feature of these devices is that all are pixelated systems, meaning that they use many small detectors rather than a single large crystal. These include positron systems (as well as standard PET tomographs, although the word pixelated is generally not used to describe them). Most pixelated cameras identify the individual detector registering a photon interaction but not the exact location of the interaction. Thus, smaller detectors mean better resolution, and the detector size in these devices is $1.6-3 \mathrm{~mm}$.

The use of a pixelated system necessitates a different method to convert the electrons resulting from radiation interactions into an electronic signal. In standard $\gamma$-cameras, the scintillation photons created in a large scintillation crystal are detected by several PMTs, using an algorithm called Anger positioning logic (after the camera's inventor, Dr. Hal Anger). The face of a modern PMT is in the neighborhood of $8 \mathrm{~mm}$ in diameter, much larger than the pixelated detectors used in these systems, requiring a different type of photon transducer.

One way to accomplish multiplication of scintillation photons is to use a position-sensitive PMT (PSPMT). Recall that a standard PMT absorbs scintillation photons at its photocathode, which releases electrons within the evacuated glass container. The electrons interact with a series of 10-12 metal dynodes, with each dynode providing a gain of 3-6 in 


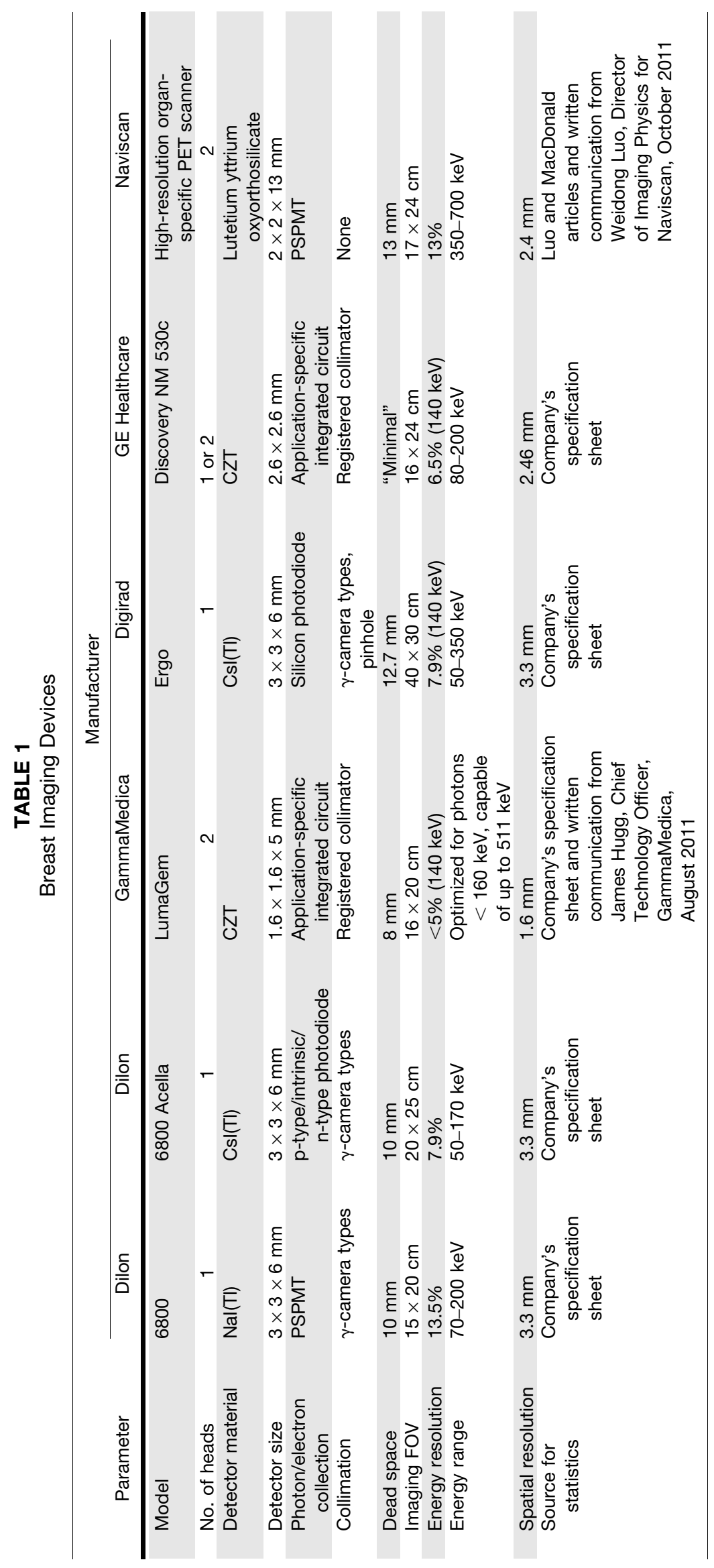


the magnitude of the electrical signal. The final electron cloud (now about a billion times larger than the original number generated at the photocathode) is collected at the terminal end of the PMT by an anode. A PSPMT similarly multiplies electrons but maintains spatial separation of the electron clouds originating at different points on the photocathode. A mesh structure with 16-20 stages of multiplication is created by layering many dynodes with guard plates perforated with holes. The anode of a PSPMT is divided into discrete pixels, each with its own connection to the downstream electronics. This system can be adapted to the pixelated system used in single-photon breast imaging devices (12).

A second technique is to replace the PMT with a solidstate device called a photodiode. A photodiode converts photons to electrons and then collects those electrons directly-much like the semiconductor radiation detectors, except that they are registering scintillation photons instead of $\gamma$-rays. They require less operating voltage than PMTs and are more rugged and compact (12). The wavelength of CsI(Tl) scintillation photons is nicely suited to these photon transducers.

The use of CZT as the detector material removes the need for a PMT completely. The output from the CZT detector element is already in the form of an electronic signal that is sent (after conversion to a digital signal and amplification) to an application-specific integrated circuit. The signal is analyzed by a pulse height analyzer, and digital information about the pixel position and timing is fed into the applicationspecific integrated circuit, which adds it into the image matrix.

\section{Collimator and Biopsy Capabilities}

The systems using $\mathrm{NaI}(\mathrm{Tl})$ or $\mathrm{CsI}(\mathrm{Tl})$ scintillation crystals use collimators similar to those of a regular $\gamma$-camera, with holes that are smaller than the individual crystals and not aligned with them. Some collimators are designed to meet the needs of both imaging of the breast, where sensitivity is quite important, and imaging of small organs, where more emphasis is placed on resolution. Slant-hole construction may be used to improve the ability to see lesions close to the chest wall. The CZT-based systems, on the other hand, use what is called a registered collimator, in which the collimator holes are square and are matched to the geometry of the detector elements. This arrangement effectively causes the detector and collimator hole to act as a single radiation detector, such that the collimator resolution determines the system resolution (13).

In the scenario of a breast tumor seen only with radionuclide imaging, we need a way to perform a biopsy of the suspect tissue based on the radionuclide image, and vendors are addressing this issue. For example, one camera has two $20^{\circ}$ slant-hole collimators, allowing views from two angles, with a grid attached above the collimator assembly. A software program identifies the grid location and depth of the lesion, based on triangulation between the views. A point source of radionuclide on the tip of the biopsy needle acts as a guide allowing the tip to be seen on an image. Biopsy devices are either available now or in clinical trials for most systems.

\section{Physical Size}

The systems being sold for breast imaging have imaging FOVs in the range of $15-20 \mathrm{~cm}$ by $20-40 \mathrm{~cm}$. Although some smaller versions are insufficient for large breasts, a $20 \times$ $25 \mathrm{~cm}$ FOV is adequate for most patients. These cameras can also be used for other nuclear medicine studies. Bone scans of extremities and thyroid scans are examples of the potential for these devices. They are generally mounted on a mammogramlike gantry, providing great flexibility for angulation and positioning.

One major drawback of standard $\gamma$-cameras for breast imaging is the large dead space around the active imaging area of the camera face. This dead space makes it impossible to isolate the breast tissue for imaging other than through prone-dependent positioning. A key advantage of the pixelated architecture is that edge effects are avoided. The devices in Table 1 all have $1 \mathrm{~cm}$ or less dead space, making possible mammogramlike imaging to include the chest wall.

\section{Compression}

As any woman who has undergone mammography knows, the worst part of the procedure is the significant amount of compression required to flatten the breast tissue. Typically, some 155-200 N (35-45 lb) of pressure are required to obtain an adequate mammographic image. The nuclear imaging devices on the market require much less pressure, generally 44-66 N (10-15 lb). This is generally tolerated without complaint (14). With single-head systems, the pressure is accomplished using a compression paddle that doubles as a radiation shield, blocking $\gamma$-rays coming from other parts of the patient's body.

Dual-head systems use the second head as the compression paddle. This has several benefits. It puts most of the breast tissue within a few centimeters (at most) of one of the detectors, improving spatial resolution. Some systems allow the separate views to be combined into a composite image, for example, a geometric mean of the views from the 2 camera heads.

\section{Positron Emission Mammography}

A device designed for coincidence imaging of the breast needs 2 heads, in order to detect coincident annihilation photons. The commercially available system uses lutetium yttrium orthosilicate crystals and PSPMTs. Each head consists of an array containing 2,028 lutetium yttrium orthosilicate crystals of $2 \times 2 \times 13 \mathrm{~mm}$ each, with an imaging area of $6 \times 16.4 \mathrm{~cm}(15)$. The 2 detectors are contained within a compression paddle and a support paddle and move in the 6-cm direction to create a FOV width of 24 $\mathrm{cm}$ (i.e., maximum FOV of $16.4 \times 24 \mathrm{~cm}$ ). Similar to other coincidence imaging systems, this system does not use absorptive collimation. Lines of response representing coincident 

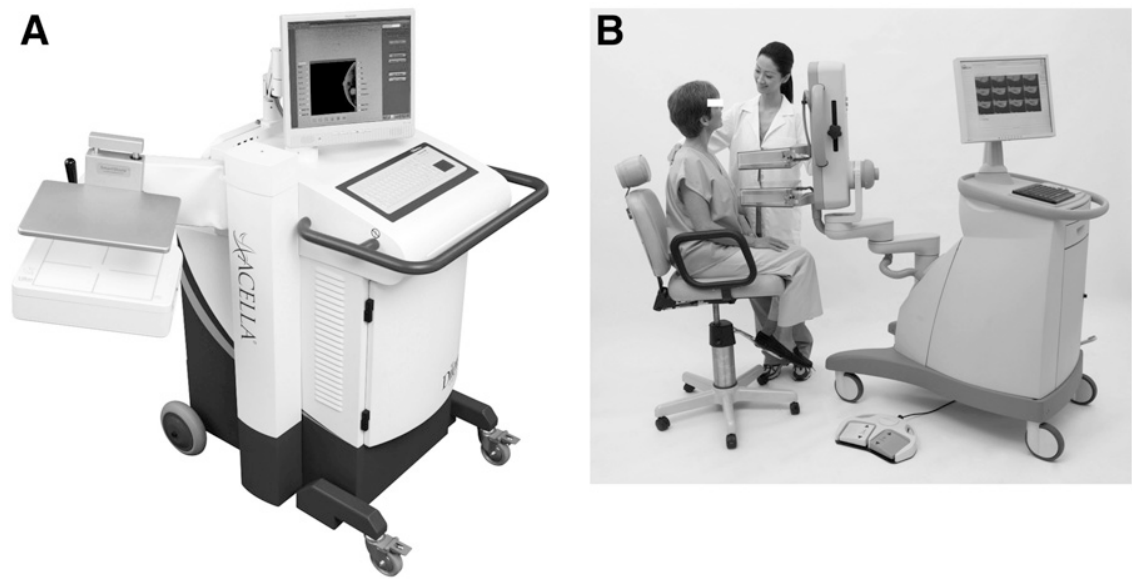

FIGURE 1. Breast imaging devices. (A) Single-photon imaging device from Dilon Diagnostics. (B) High-resolution PET scanner from Naviscan. In both systems, detectors are mounted on mammographic gantries, allowing for range of motion and positioning in either sitting or standing position. Each system also has small footprint. photons are stored as list-mode data, and reconstruction uses a 3-dimensional maximum-likelihood expectation maximization algorithm. The reconstruction produces a total of 12 or 24 image planes. Spatial resolution is measured at 1.8 and $2.4 \mathrm{~mm}$ in standard and high-resolution modes, respectively $(15,16)$.

Another system uses avalanche photodiodes on both sides of $2 \times 2 \mathrm{~mm}$ lutetium yttrium orthosilicate crystals (17). This arrangement allows for depth-of-interaction determinations for each detected coincidence, which improves spatial resolution to $1.2 \mathrm{~mm}$. Other devices using different scintillators and electronic configurations are in development (18). Because corrections for dead time, attenuation, and scatter are not applied in all systems, quantitative estimation of lesion activity with these systems may be less accurate than similar determinations obtained from standard PET tomographs (19).

\section{BREAST IMAGING TECHNIQUES}

Breast imaging in nuclear medicine goes under several names. In the 1990s, when prone-dependent imaging with standard $\gamma$-cameras was done, it was called scintimammography. With the advent of small imaging devices designed for breast imaging, developers came up with new descriptions: molecular breast imaging and breast-specific gamma imaging. Positron imaging of the breast goes by the name positron emission mammography.

To understand the operation of nuclear breast imaging devices, we need a little understanding of mammography. Standard views are the craniocaudal view, in which x-rays pass through the breast in a superior-to-inferior direction, and the mediolateral oblique view, in which the x-ray tube is positioned in the middle of the body and the film laterally and inferiorly to the breast (20). Although mammographers have an assortment of magnifications and additional views, these 2 standard views will be the major ones used in nuclear breast imaging.

Figure 1 shows 2 examples of these imagers, one for single-photon imaging and one for positron emission mam- mography. Figure 2 illustrates the craniocaudal, mediolateral oblique, and axillary imaging positions with a single-head device. Figure 3 is an example case showing a malignant lesion with sestamibi, and Figure 4 illustrates the tomographic views obtained with ${ }^{18} \mathrm{~F}-\mathrm{FDG}$ and the positron device. All images and photographs were supplied by the commercial vendors.

The question of positioning brings up a point of controversy: who should be the imaging technologist? Although the procedure is clearly a nuclear medicine technique, nuclear medicine technologists are not nearly as adept at positioning breasts as mammographers. In some sites, the nuclear medicine technologist performs the injection, but a mammographer is responsible for acquiring the images. Some vendors recommend that the mammographer who obtained the mammography films also position for the nuclear procedure. Location of the device (hospital or large-scale imaging clinic vs. breast imaging center) plays a role, as do state regulations in some instances. Perhaps the most logical solution is that nuclear medicine technologists perform these procedures after receiving training from mammographers on how to obtain high-quality breast images. Keep in mind that technologist radiation exposure, especially to the hands, will be high due to the need to manipulate the breast to obtain good images.

One site's procedure for imaging with ${ }^{99 \mathrm{~m}} \mathrm{Tc}$-sestamibi has been described in an article in this journal (21). Key points in the procedure are as follows:

- Injection is via an indwelling catheter in the contralateral arm to the breast in question, if known. It is important to avoid infiltration and any possibility of lymphatic or vascular trapping.

- Imaging commences soon after injection, according to the article (21); others (including the Society of $\mathrm{Nu}$ clear Medicine procedure guideline (22)) recommend waiting 5-10 min.

- Images are acquired for 5-10 min each, such that the total imaging time is about an hour if all 4 standard views are taken. 

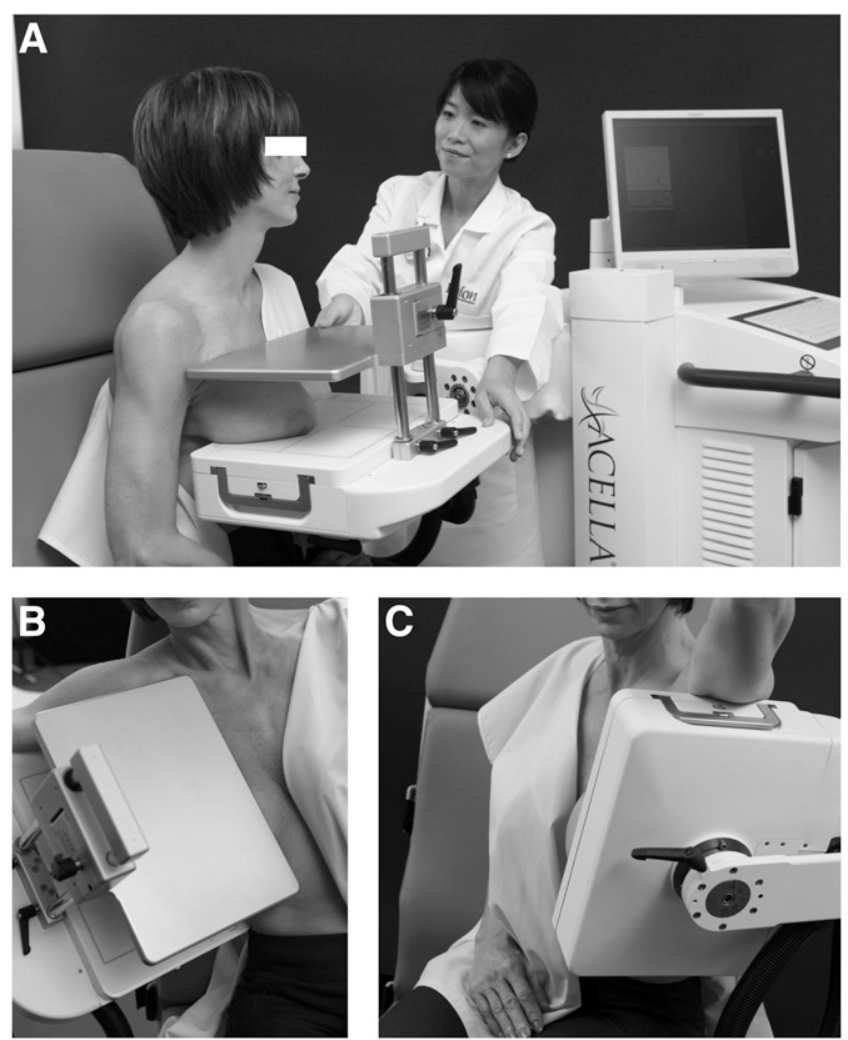

FIGURE 2. Photographs showing positioning for craniocaudal $(\mathrm{A})$, mediolateral oblique $(\mathrm{B})$, and axillary $(\mathrm{C})$ breast imaging views. System shown has single detector head and compression paddle. (Courtesy of Dilon Diagnostics.)

- Patients are usually seated for the images (most manufacturers allow for standing views as well). The camera heads on most systems can be placed at a variety of angles and heights.

- The mediolateral oblique views should be obtained at an angle parallel to the pectoralis muscle and showing it if possible. Nuclear medicine technologists will want to review this anatomy to visualize the location of this muscle.

The article (21) discusses additional views used in the authors' institution.

For imaging with ${ }^{99 \mathrm{~m}} \mathrm{Tc}$-sestamibi, it is recommended that nuclear breast imaging be performed within the first 2 wk after a woman's menstrual cycle and several weeks after an invasive procedure such as a biopsy or cyst aspiration (22). These recommendations are similar to those for breast ultrasound and MRI studies. Later in the menstrual cycle, uptake of sestamibi may be diffuse and patchy, making image interpretation more difficult (23).

The question of dosage for ${ }^{99 \mathrm{~m}} \mathrm{Tc}$-sestamibi is currently controversial. The package insert lists $740-1,110 \mathrm{MBq}$ (20-30 $\mathrm{mCi}$ ) as appropriate for breast imaging, but this was the dosage used for the prone-dependent imaging. Technically, administering something less than the amount recommended in the package insert constitutes an off-label use. But breast-specific devices are able to get better images with less radioactivity; researchers have shown good results with $296 \mathrm{MBq}(8 \mathrm{mCi})$ and aim to reduce this to $148 \mathrm{MBq}$ (4 mCi) or even $74 \mathrm{MBq}(2 \mathrm{mCi})$. At $74 \mathrm{MBq}$, the effective dose from a nuclear breast study is $0.7 \mathrm{mSv}$, approximately the same as that from mammography $(0.1-0.6 \mathrm{mSv})(24)$. The additional effective dose from the breast imaging procedure will be important as public concern about medical radiation exposure continues, especially since the alternative imaging modalities (ultrasound and MRI) do not involve radiation exposure.

Positron emission mammography is approached a little differently. Many institutions using this technique are doing so in conjunction with a whole-body ${ }^{18}$ F-FDG PET scan. Because most of these patients already have known or suspected spread of their cancer, the whole-body scan is necessary for staging. The patient undergoes the whole-body scan at the institution's normal uptake time (usually 45-60 min) and then follows with the positron emission mammography imaging. Because an ${ }^{18} \mathrm{~F}$-FDG scan has an effective dose of 5-8 mSv, the scan ideally should be obtained only on patients who have a need for the whole-body scan. Advantages of breast imaging with ${ }^{18} \mathrm{~F}-\mathrm{FDG}$ include the fact that it is not affected by hormonal status relative to the menstrual cycle (25), as well as its increased target-to-background ratio, compared with ${ }^{99 \mathrm{~m}} \mathrm{Tc}-$ sestamibi (oral communication, Judith Kalinyak, Society of Nuclear Medicine annual meeting, June 8, 2010).

Breast imaging devices, like other radiation detection instruments, require quality control. In most cases, this amounts to a daily flood scan using a ${ }^{57} \mathrm{Co}$ sheet source that can be completed in 5-10 min, and a monthly or quarterly calibration procedure that takes $20-30 \mathrm{~min}$. Manufacturers provide quality assurance protocols for each system. The quality control for the PET device referenced in Table 1 includes a daily acquisition to evaluate system efficiency and a monthly update of correction tables and monitoring of the electronics.

\section{NUCLEAR IMAGING IN THE SCHEME OF BREAST CANCER DETECTION}

Several indications for breast scintigraphy are listed in the Society of Nuclear Medicine Procedure Guideline (22). These include patients with recently detected breast malignancies, to evaluate the extent of disease; patients at high risk for breast malignancy; patients with indeterminate abnormalities or diagnostic concerns on mammography; patients with technically difficult breast imaging; patients for whom breast MRI would be indicated but who cannot (or will not) undergo MRI; and patients undergoing preoperative chemotherapy, to monitor their progress.

Mammography results are categorized using the Breast Imaging Reporting and Data System (BI-RADS), under the auspices of the American College of Radiology. Using this system, additional follow-up (including breast scintigraphy) is recommended in persons with BI-RADS scores of 3, 4, or 5 (20).

Mammography will always be the first option in the initial diagnosis of breast cancer. Other modalities most often come into play after a suggestive lesion has been seen 
A
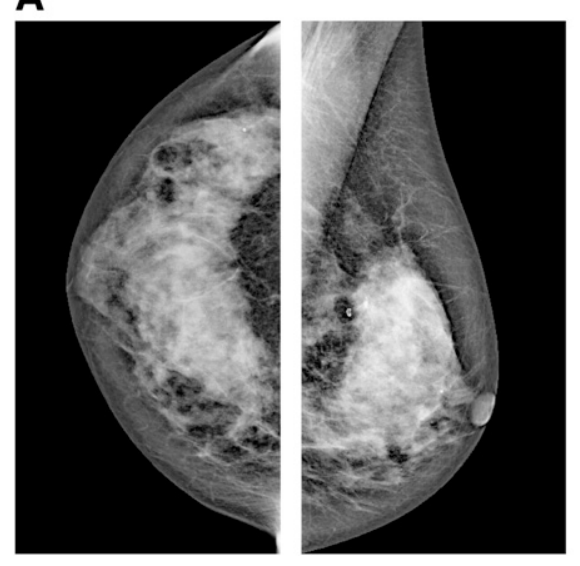

B

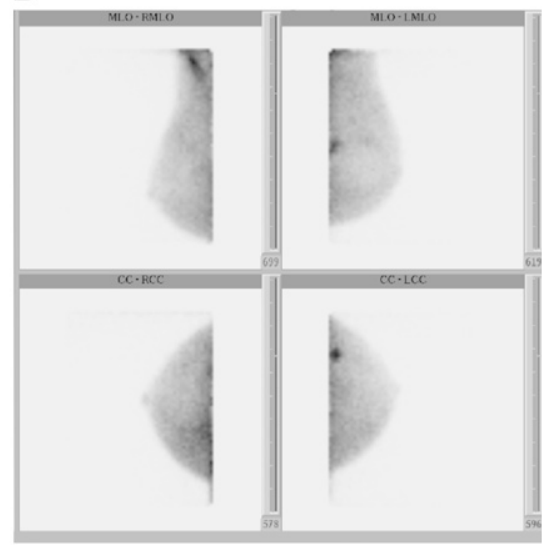

FIGURE 3. Breast cancer seen with 99mTc-sestamibi. (A) Craniocaudal and mediolateral oblique mammographic views of left breast in patient with dense breasts. Suggestive area corresponded to location of prior biopsy that documented benign lesion. (B) Nuclear images show uptake in area of prior biopsy, which was determined to be infiltrating lobular carcinoma. (Courtesy of Dilon Diagnostics.) but before an invasive biopsy has been done. In this context, the chief disadvantage of MRI and ultrasound is their low specificity (2); therefore, nuclear breast imaging is becoming an increasingly important adjunct (26).

Evidence of the utility of nuclear breast imaging is accumulating. Several studies show the benefit of nuclear imaging in sorting out mammographic findings $(14,23,25$, $26,27)$. In a variety of patient populations and diagnostic situations, sestamibi and ${ }^{18}$ F-FDG imaging using breastspecific devices have sensitivity in the 85\%-95\% range and specificity in the $60 \%-95 \%$ range. These numbers compare favorably with the values for MRI and sonography, the competing secondary imaging modalities. Further, in patients with newly diagnosed breast cancer, nuclear breast imaging can demonstrate additional abnormalities and may change patient management (28).

A particular subset of patients for whom nuclear breast imaging makes a great deal of sense is women with dense breasts. The overall incidence of dense breasts is high: up to one third of women over $50 \mathrm{y}$ old and half of those under $50 \mathrm{y}$ old (27). Dense breast tissue is by itself a predictive factor for breast cancer: women with dense breasts have a greater than 3 times increased likelihood for breast cancer, according to a recent study (29). And the sensitivity of mammography for cancerous lesions is decreased in women with dense breast tissue. Dense breast tissue is "the factor most closely associated with failure to detect breast cancer by mammography" (27). This would seem to be an obvious place to begin in terms of building a case for nuclear breast imaging.

Another group of patients who would benefit from nuclear breast imaging is those with an initial finding of a lesion assigned a BI-RADS score of 3. This score indicates a lesion that is probably benign, but short-term follow-up is needed to confirm that it is not changing over time. Many women do not undergo repeated mammography in 6 mo as recommended by the radiologist. If nuclear breast imaging is readily available, patients could be asked to stay for a molecular breast study. Given the high sensitivity and specificity of this technique, most of these BI-RADS 3 findings could be identified as either likely malignant or likely benign, improving outcomes and reducing anxiety.
A

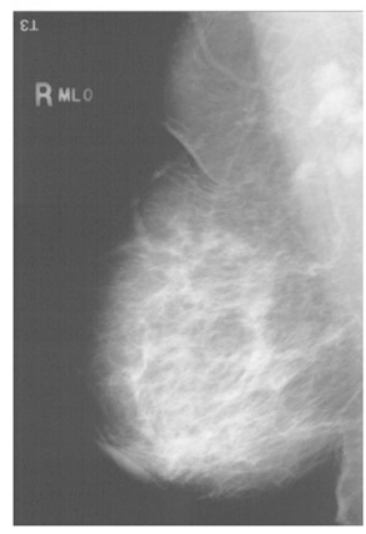

B

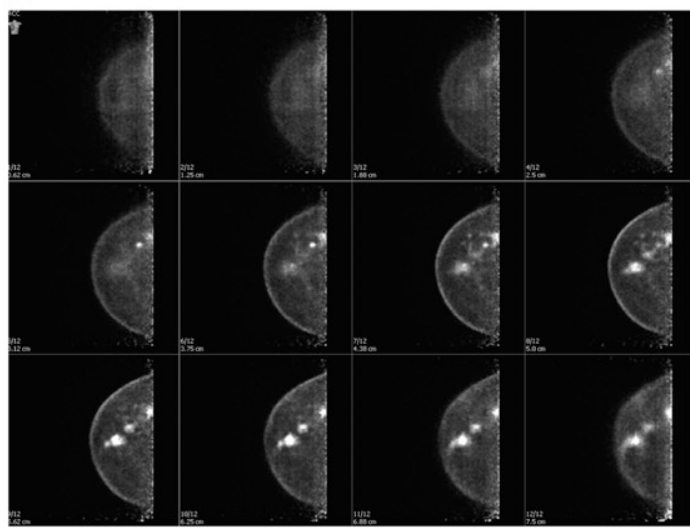

C

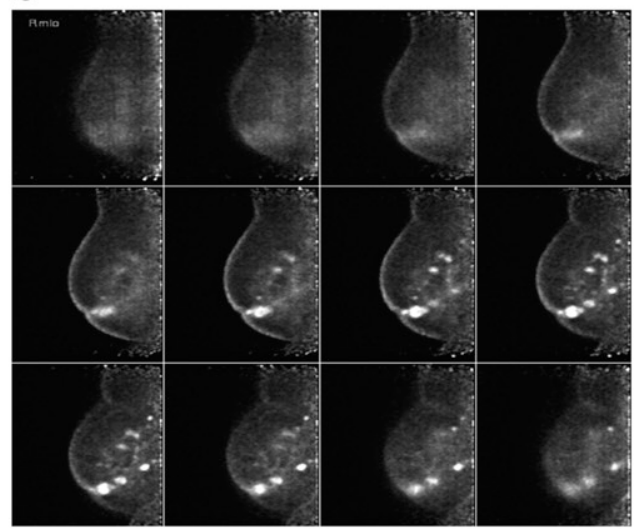

FIGURE 4. Tomographic slices from ${ }^{18}$ F-FDG breast study. Mediolateral oblique (B) and craniocaudal (C) views of multifocal cancer in right breast are shown, along with corresponding right mediolateral oblique mammogram (A). Slice thickness depends on distance between the 2 detectors, since this system always produces either 12-slice or 24-slice study. (Obtained from East Jefferson General Hospital, Metairie, Louisiana, and provided courtesy of Naviscan, Inc.) 


\section{CONCLUSION}

Nuclear breast imaging has been in existence for $20 \mathrm{y}$ or more but has become more popular over the last $5 \mathrm{y}$ or so. The major reason is the development of breast-specific imaging devices. Both the variety of commercially available devices and the number of installations are rising. Articles document the advantages and utility of sestamibi and ${ }^{18} \mathrm{~F}$ FDG in the diagnosis of breast cancer, in several situations.

Nuclear imaging of the breast has significant advantages over its main competitor, MRI. MRI is considerably more expensive than a ${ }^{99 \mathrm{~m}} \mathrm{Tc}-$ sestamibi breast study. In addition, MRI is contraindicated in persons with metal implants of any kind, and people with claustrophobia often refuse to have the scan done. Nuclear breast devices described in this article overcome both of these problems and have the added advantage of providing views equivalent to mammography, facilitating the correlation between the two. And most importantly, the specificity of nuclear breast imaging is significantly higher than that of MRI, while maintaining good sensitivity.

The key to effective nuclear imaging of the breast is the use of devices optimized for breast imaging. Development of these imagers, although still ongoing, appears to have reached a level of maturity compatible with clinical adoption. Technologists will need to learn how to manipulate the breast to obtain high-quality images, and the issue of ${ }^{99 \mathrm{~m}} \mathrm{Tc}$-sestamibi dosage is as yet unresolved. The case for nuclear breast imaging will need to be made, both to breast radiologists and to oncologists. With these efforts, nuclear breast imaging has the potential to play a major role in improving the diagnosis of breast cancer.

\section{ACKNOWLEDGMENT}

No potential conflict of interest relevant to this article was reported.

\section{REFERENCES}

1. Taillefer R. Clinical applications of ${ }^{99 \mathrm{~m}} \mathrm{Tc}$-sestamibi scintimammography. Semin Nucl Med. 2005;35:100-115.

2. Swart R. Breast cancer screening. Medscape Reference Web site. Available at: http:// emedicine.medscape.com/article/1945498-overview. Accessed April 19, 2012.

3. Berg WA, Blume JD, Adams AM, et al. Reasons women at elevated risk of breast cancer refuse breast MR screening: ACRIN 6666. Radiology. 2010;254:79-87.

4. Aktolun C, Bayhan H, Kir M. Clinical experience with Tc-99m MIBI imaging in patients with malignant tumors: preliminary results and comparison with Tl-201. Clin Nucl Med. 1992;17:171-176.
5. Khalkhali I, Mena I, Jouanne E, et al. Prone scintimammography in patients with suspicion of carcinoma of the breast. J Am Coll Surg. 1994;178:491-497.

6. Lee JH, Rosen EL, Mankoff DA. The role of radiotracer imaging in the diagnosis and management of patients with breast cancer: part 1 -overview, detection, and staging. J Nucl Med. 2009;50:569-581.

7. Lee JH, Rosen EL, Mankoff DA. The role of radiotracer imaging in the diagnosis and management of patients with breast cancer: part 2-response to therapy, other indications, and future directions. J Nucl Med. 2009;50:738-748.

8. Bowen SL, Wu Y, Chaudhari AJ, et al. Initial characterization of a dedicated breast PET/CT scanner during human imaging. J Nucl Med. 2009;50:1401-1408.

9. Knoll GF. Radiation Detection and Measurement. 3rd ed. New York, NY: John Wiley and Sons, Inc; 2000:235, 238.

10. Hruska CB, O'Connor MK. CZT detectors: how important is energy resolution for nuclear breast imaging? Phys Med. 2006;21(suppl 1):72-75.

11. Heller S, Zanzonico P. Nuclear probes and intraoperative gamma cameras. Semin Nucl Med. 2011;41:166-181.

12. Madsen MT. Recent advances in SPECT imaging. J Nucl Med. 2007;48:661-673.

13. Mueller B, O'Connor MK, Blevis I, et al. Evaluation of a small cadmium zinc telluride detector for scintimammography. J Nucl Med. 2003;44:602-609.

14. Rhodes DJ, O'Connor MK, Phillips SW, et al. Molecular breast imaging: a new technique using technetium Tc-99m scintimammography to detect small tumors of the breast. Mayo Clin Proc. 2005;80:24-30.

15. Luo W, Anaskin E, Matthews CC. Performance evaluation of a PEM scanner using the NEMA NU 4-2008 small animal PET standards. IEEE Trans Nucl Sci. 2010;57:94-103.

16. MacDonald L, Edwards J, Lewellen T, et al. Clinical imaging characteristics of the positron emission mammography camera: PEM Flex Solo II. J Nucl Med. 2009;50:1666-1675.

17. Neves JA. The ClearPEM breast imaging scanner. Nucl Instr Meth A. 2011;628: 444-447.

18. Moadel RM. Breast cancer imaging devices. Semin Nucl Med. 2011;41:229-241.

19. Springer A, Mawlawi OR. Evaluation of the quantitative accuracy of a commercially available positron emission mammography scanner. Med Phys. 2011;38:2132-2139.

20. Dongola N. Mammography in breast cancer. Medscape Reference Web site. Available at: http://emedicine.medscape.com/article/346529-overview. Accessed April 19, 2012.

21. Jones EA, Phan TD, Blanchard DA, Miley A. Breast-specific gamma imaging: molecular imaging of the breast using ${ }^{99 \mathrm{~m} T c-s e s t a m i b i}$ and a small-field-of-view gamma camera. J Nucl Med Technol. 2009;37:201-205.

22. The SNM procedure guideline for breast scintigraphy with breast-specific $\gamma$-cameras 1.0. Society of Nuclear Medicine Web site. Available at: http://interactive.snm. org/docs/BreastScintigraphyGuideline_V1.0.pdf. Accessed April 19, 2012.

23. O'Connor M, Rhodes D, Hruska C. Molecular breast imaging. Expert Rev Anticancer Ther. 2009;9:1073-1080.

24. Mettler FA, Huda W, Yoshizumi TT, Mahesh M. Effective doses in radiology and diagnostic nuclear medicine: a catalog. Radiology. 2008;248:254-263.

25. Ferrara A. Nuclear imaging in breast cancer. Radiol Technol. 2010;81:233-246.

26. Brem RF, Fishman M, Rapelyea JA. Detection of ductal carcinoma in situ with mammography, breast specific gamma imaging, and magnetic resonance imaging: a comparative study. Acad Radiol. 2007;14:945-950.

27. Rhodes DJ, Hruska CB, Phillips SW, Whaley DH, O'Connor MK. Dedicated dual-head gamma imaging for breast cancer screening in women with mammographically dense breasts. Radiology. 2011;258:106-118.

28. Killelea BK, Gillego A, Kirstein LJ, et al. George Peters award: how does breastspecific gamma imaging affect the management of patients with newly diagnosed breast cancer? Am J Surg. 2009;198:470-474.

29. Yaghjyan L, Colditz GA, Collins LC, et al. Mammographic breast density and subsequent risk of breast cancer in postmenopausal women according to tumor characteristics. J Natl Cancer Inst. 2011;103:1179-1189. 